\title{
Simulation of the GEM detector for BM@N experiment
}

\author{
Dmitriy Baranov ${ }^{1, \star}$ and Oleg Rogachevsky ${ }^{1, \star \star}$ \\ ${ }^{1}$ Joint Institute for Nuclear Research, 6 Joliot Curie St., Dubna, Moscow region, 141980 Russia
}

\begin{abstract}
The Gas Electron Multiplier (GEM) detector is one of the basic parts of the BM@N experiment included in the NICA project. The simulation model that takes into account features of signal generation process in an ionization GEM chamber is presented in this article. Proper parameters for the simulation were extracted from data retrieved with the help of Garfield++ (a toolkit for the detailed simulation of particle detectors). Due to this, we are able to generate clusters in layers of the micro-strip readout that correspond to clusters retrieved from a real physics experiment.
\end{abstract}

\section{Introduction}

The BM@ $\mathrm{N}^{1}[1]$ is one of the first heavy-ion experiments of the $\mathrm{NICA}^{2}$ project $[2,3]$ at the Joint Institute for Nuclear Research (JINR) in Dubna. The main distinctive feature of this experiment is an available facility to accelerate heavy ions to an energy between 2 and $6 \mathrm{~A} \mathrm{GeV}$. Such energy range covers the region of the maximum baryon density. Thus the BM@N experiment with a fixed target gives the great ability to study baryonic matter on relativistic heavy ion collisions at the Nuclotron.

The BM@N setup consists of a tracking system, a time of flight system for particle identification and some detectors to measure event characteristics.

\section{BM@N GEM tracker}

The BM@N central tracking system is comprised of GEM chambers with two-coordinate micro-strip readout [4]. Each GEM chamber registers the ionization trail left by charged particle in the sensitive gas volume. The overall structure of the detector is a set of separated GEM stations that are located along the beam-axis at a certain distance from each other. Full GEM tracker configuration (figure 1) includes 12 such stations, each of these has a certain type of the GEM chamber.

GEM plane is divided into areas. The areas called "hot zones" are generally placed at the center of the station. Thus we have parts with strip readout electrically independent from each other in one chamber. It allows us to process high multiplicity events.

\footnotetext{
$\star^{\star}$ e-mail: dbaranov@jinr.ru

$\star \star$ e-mail: rogachevsky@jinr.ru

${ }^{1}$ Baryonic Matter at Nuclotron

${ }^{2}$ Nuclotron Based Ion Collider fAcility
} 


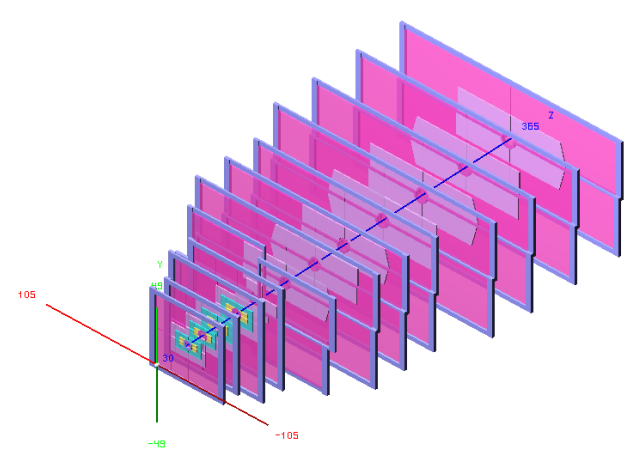

Figure 1. BM@N GEM tracker: full configuration.

\subsection{Gas Electron Multiplier in BM@N}

There are used triple-GEM chambers in the inner tracking detector for the BM@N experiment. Chamber capacity is filled with a gas mixture of $\mathrm{Ar}$ and $\mathrm{CO}_{2}$ in the ratio 70:30. Each triple-GEM has multi-layer structure (figure 2): a drift gap, where multiple ionization processes happen, two transfer gaps, where drifted electrons passing through the GEM foils are multiplied by impact ionization in high electric inside foil holes, and an induction gap, where produced electron avalanches drift to the readout plane. The signal registered by readout strips from one charged particle has a cluster structure.

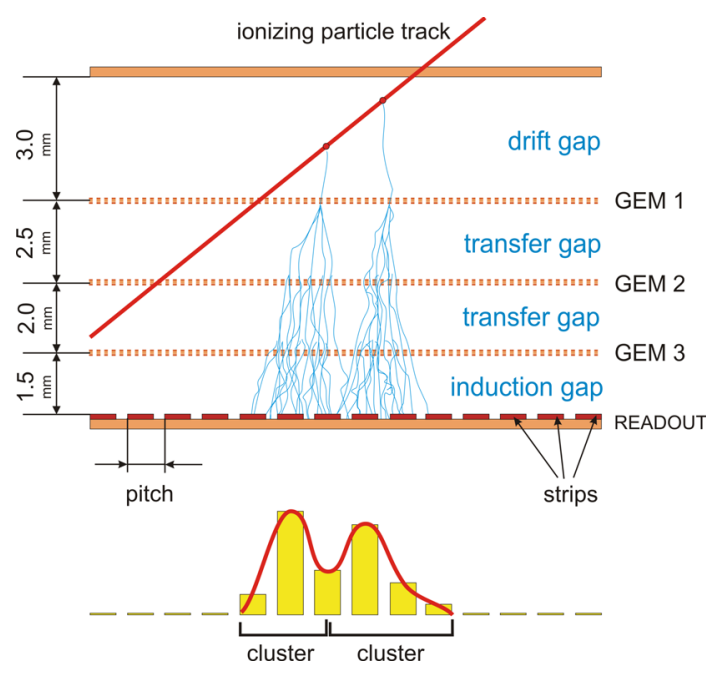

Figure 2. BM@N triple-GEM structure and signal formation scheme.

More detailed information about working principles and signal formation in GEM detectors are described in the works $[5,6]$. 


\section{Simulation model}

In order to obtain proper parameters for the strip cluster generation (such as mean shift of electrons in magnetic field, electron diffusion and gas ionization characteristics) we used the Garfield++ toolkit [7].

Since the magnetic field inside the magnet is directed perpendicular to electric field inside the GEM, produced electron avalanches are shifted from its initial positions by Lorentz force. The dependence of the mean electron shift in the transverse drift direction on its traversed distance for different GEM gaps is shown below (figure 3, left). The dependence of the mean electron shift on the distance to the readout plane for various magnetic field strength is also shown (figure 3, right).
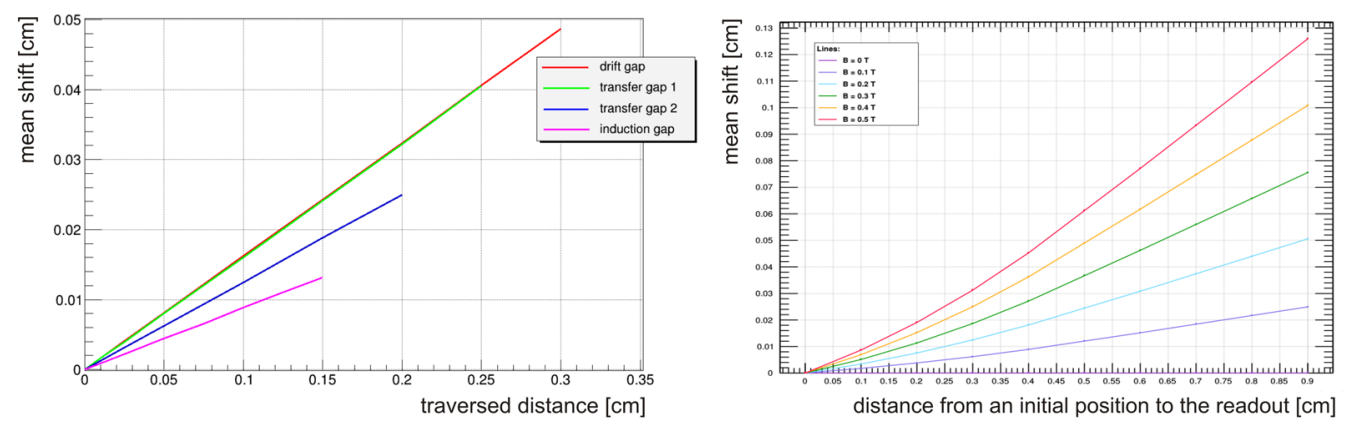

Figure 3. (Left) Dependence of the mean electron shift in the transverse drift direction on its traversed distance for all GEM gaps; (right) dependence of the mean electron shift on the distance to the readout plane for various magnetic field strength.

An electron cloud drifting towards the anode along field lines in electric field spreads due to random thermal motion and repulsion. Electron diffusion affects the size of complete electron avalanches that induce output signal on the readout plane. The graph below shows the dependence of the standard deviation (sigma) of electrons in the transverse drift direction on its traversed distance for all GEM gaps (figure 4, left). The cumulative distribution (on the readout plane) of electrons drifted through the thickness of the GEM chamber $(0.9 \mathrm{~cm})$ taking into account the electron shift and electron diffusion is also shown (figure 4, right).

We also obtained ionization characteristics of our gas media for different types of charged particles. The graph below presents an example of the distribution of primary electron-ion clusters along $1 \mathrm{~cm}$ track for particle pi+ with initial momentum $1 \mathrm{GeV} / \mathrm{c}$ (figure 5, left). In the other graph you can observe the distribution of collision distances between the electron-ion clusters for the same particle (figure 5, right).

Entire simulation model of BM@N GEM tracker includes the geometric model implemented as a ROOT [8] geometry file and the program logic model to generate and process output data. The geometric model describes the spatial dimensions and material properties of the basic detector elements. This model is used to simulate the passage of particles through the detecting planes with Geant 4 software [9] based on Monte Carlo methods. The MC-points, as the result of Geant4 production, allow us to generate output data (strip clusters) that correspond to the real data.

The following data transformation has several steps:

- From MC-point (obtained by the Geant4 simulation engine) we have parameters, such as: an initial position of the entry point, momentum and type of a particle; 

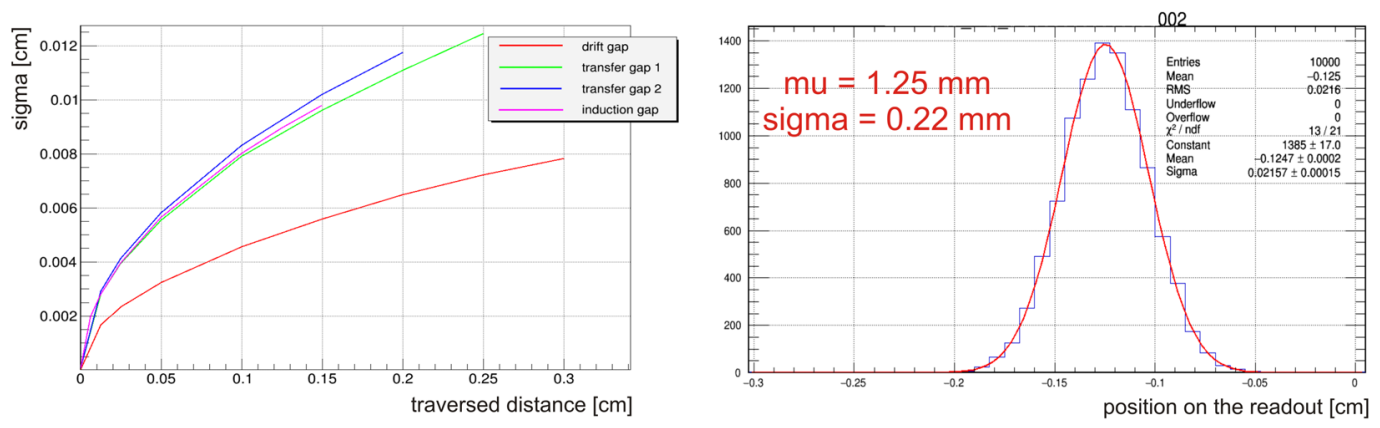

Figure 4. (Left) Dependence of the standard deviation (sigma) of electrons in the transverse drift direction on its traversed distance for all GEM gaps; (right) density distribution of electrons passed through the thickness of the GEM chamber $(0.9 \mathrm{~cm})$.
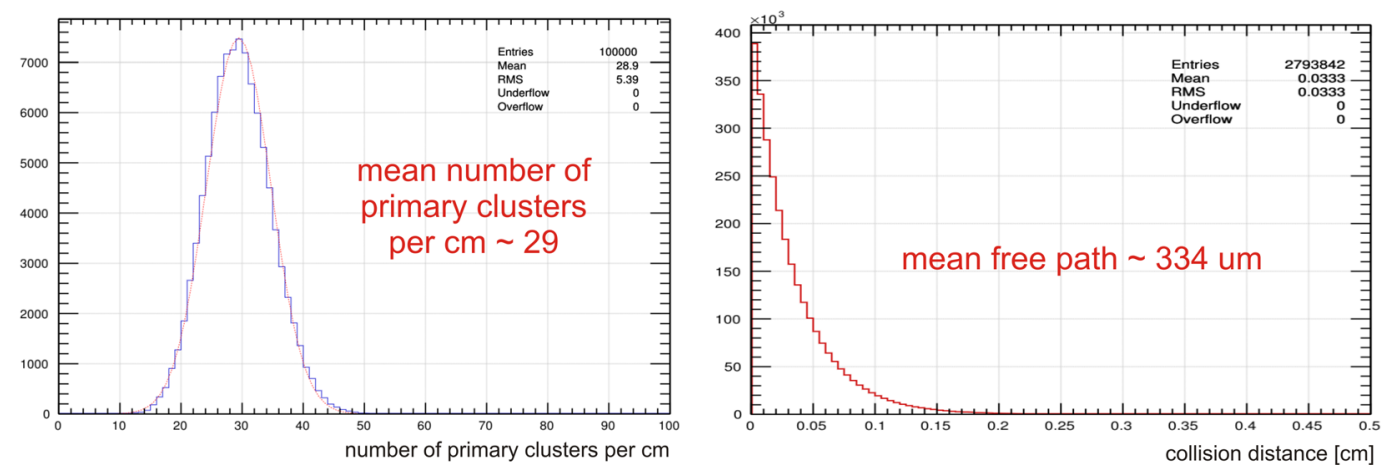

Figure 5. (Left) Distribution of primary electron-ion clusters along $1 \mathrm{~cm}$ of the track for the particle pi+ with initial momentum $1 \mathrm{GeV} / \mathrm{c}$; (right) distribution of collision distances for the particle pi+ with initial momentum $1 \mathrm{GeV} / \mathrm{c}$.

- Further, define a particle track through the GEM volume;

- Due to distributions of ionization parameters for the current particle we determine size and position of electron clusters along the track;

- According to dependencies of the mean electron displacement and standard deviation we generate electrons in each layer to calculate the strip signal.

The proposed algorithm allows to reduce considerably the time of data simulation in comparison to the full microsimulation by means of the Garfield++. Such performance is achieved by excluding non-essential details that have a little effect on the resulting signal, but take too much time to process.

Thus the output data received with our simulation model of the BM@N GEM tracker take into account features of the real signal formation in the GEM chamber. Relying on these data we can get preliminary evaluation of detector efficiency for various multiplicity events. The figure beneath (figure 6) demonstrates an example of a strip cluster registered by the readout in the model for the particle track with 15 degrees inclination angle from the beam axis. 

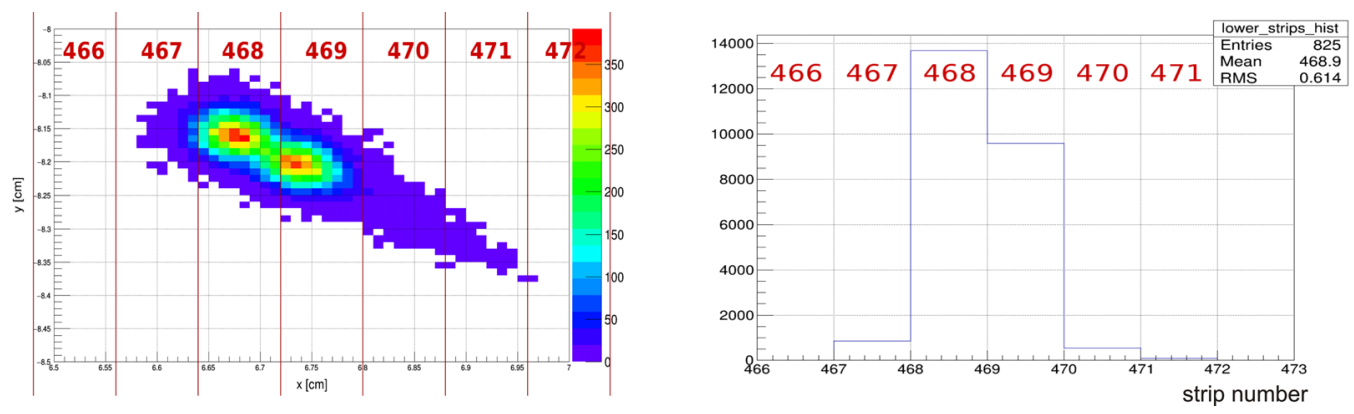

Figure 6. (Left) Cluster on the readout plane; (right) strip cluster signal.

The signal values on the strips are in abstract units, because subsequent processing steps do not depend on specific measurement units.

Simulation and subsequent reconstruction of the signal using the model was carried out to estimate model adequacy basing on sufficient statistics. The resulting accuracy of coordinate reconstruction can be evaluated on the distribution of residuals for both coordinates (figure 7).
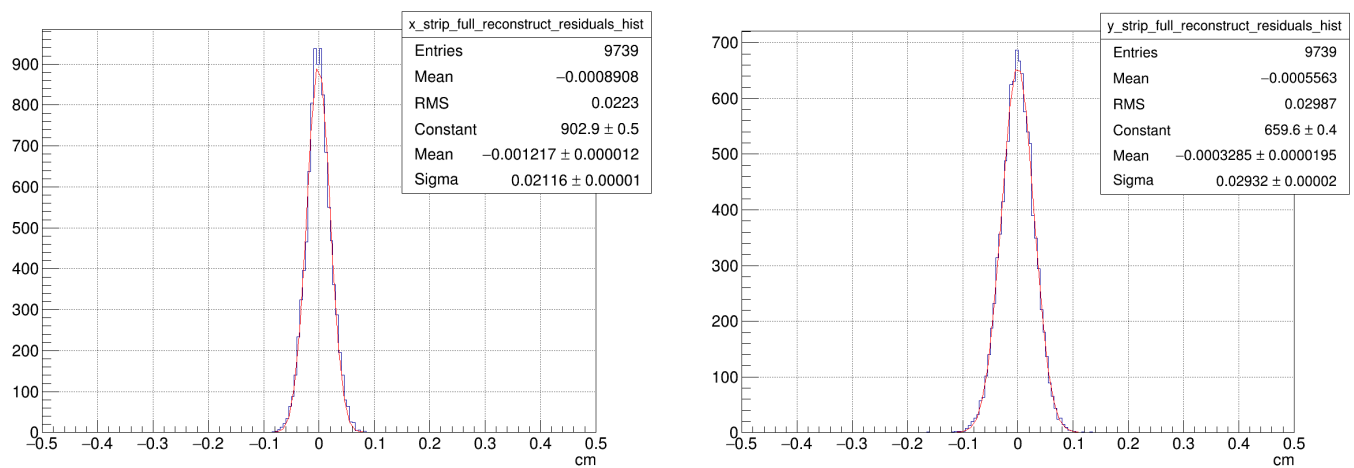

Figure 7. (Left) Residual error of X-reconstructed coordinate; (right) residual error of Y-reconstructed coordinate. The results are presented for particle tracks with inclination angle from 0 to 15 degrees from beam axis.

\section{Conclusion}

The algorithm described in the article allows to generate data equivalent to realistic. The detailed simulation of physics processes occurring in the GEM chamber to form the output signal can be carried out with Garfield++ toolkit. However this approach is unacceptable for high multiplicity events because of the huge computation time. Therefore, this toolkit was used for extracting parameters required to develop our model. Presented method takes into account factors such as particle track inclination and magnetic field influence. 


\section{References}

[1] M. Kapishin (BM@ N), Eur. Phys. J. A 52, 213 (2016)

[2] V. Kekelidze et al., Nucl. Phys. A 956, 846 (2016)

[3] The NICA Complex, http://nica.jinr.ru

[4] M. Ziegler et al., Nucl. Instrum. Meth. A 471, 260 (2000)

[5] F. Sauli, Nuclear Instruments and Methods in Physics Research Section A: Accelerators, Spectrometers, Detectors and Associated Equipment 805, 2 (2016)

[6] R. Bouclier et al., IEEE Transactions on Nuclear Science 44, 646 (1997)

[7] Garfield++, http://garfieldpp.web.cern.ch

[8] ROOT, https://root.cern.ch

[9] Geant4, http://geant4.cern.ch 